\title{
Creep Life Uncertainty Assessment of a Gas Turbine Airfoil
}

\author{
Mr. Jon M. Wallace* and Dr. Dimitri N. Mavris ${ }^{\dagger}$ \\ Aerospace Systems Design Laboratory \\ School of Aerospace Engineering \\ Georgia Institute of Technology \\ Atlanta, GA 30332-0150
}

\begin{abstract}
Hot gas path turbine components are exposed to very severe and complex boundary conditions and many other sources of variation during their design, production, and operation. Consequently, the useful life of these components can exhibit considerable scatter. A complex, multi-physics environment has been created to automate the bulk creep life assessment of a gas turbine airfoil for a land-based, heavy duty power generation unit. An uncertainty assessment using the combined response surface Monte Carlo method is conducted with the developed environment. Results of this study are given and found to be in agreement with a more theoretical solution using power series approximation. Some widely used assumptions in conducting component level reliability assessments are investigated and discussed.
\end{abstract}

\section{Introduction}

Given that components of systems such as gas turbines can show considerable scatter in their actual lives, methods are sought and have been applied to quantify this uncertainty. One approach to predicting such dispersion is to combine probabilistic analysis with deterministic simulations. Thus, existing computer simulation models are utilized without requiring newly derived and implemented probabilistic models. However, accurate deterministic predictions of the life of such components can involve tremendous computational and personnel resources. Further complicating this is the fact that probabilistic analyses increase the dimensionality of the problem, which can easily become prohibitive given the already time-consuming deterministic analysis. Fortunately, several probabilistic methods exist, which show promise in providing efficient probabilistic results for components requiring longrunning deterministic analyses. These include approximation methods, such as statistical response surface, analytical sensitivity-based methods, as well as variance reduction techniques (VRT) based on Monte Carlo simulation. Haldar and Mahadevan $(2000)^{1}$, among others, have given a thorough summary of the methods currently used.

The objective of this study was to conduct an uncertainty assessment of a nominal gas turbine airfoil using the statistical response surface approximation method and Monte Carlo simulation. The multi-disciplinary life assessment environment used for predicting the gas turbine airfoil life is described including a sophisticated computational environment created to automate this process. An overview of the response surface method coupled with Monte Carlo simulation is given. Results of the study are examined and several comparisons made.

\section{Life Assessment Environment}

A sophisticated creep life simulation environment for a landbased, gas-turbine engine turbine bucket has been developed. The component selected is a mid-stage turbine bucket from a heavy-duty gas turbine engine. The bucket material is a cast and equiaxed, nickel-based super-alloy. This particular bucket design includes eight radial cooling holes supplied by compressor bleed air.

Several mechanisms can contribute to the failure of such a component. Examples of such failure mechanisms include low cycle fatigue, creep, oxidation, overstress, and fatigue crack growth leading to fracture. For the purpose of demonstrating the method pursued, only the creep life response is considered and is done so in a section average sense at various airfoil sections of interest (see Figure 1).

The calculation of the bucket creep life requires the integration of numerous complex analyses using multiple variables. The structure of the analysis steps and data flow for the bucket creep life analysis is depicted in Figure 2. The analyses parameterized by the environment are identified by the dashed line. They include a preliminary external boundary condition

\footnotetext{
* Graduate Research Assistant, Aerospace Systems Design Laboratory (ASDL), Georgia Tech, Member AIAA

${ }^{\dagger}$ Boeing Chair for Advanced Design, School of Aerospace Engineering, Director ASDL, Senior Member AIAA Copyright $@$ by Jon M. Wallace and Dimitri N. Mavris. Published by the American Institute of Aeronautics and Astronautics, Inc. with permission.
} 
analysis, cooling-hole analysis, thermal solid analysis, mechanical analysis, and finally a creep life analysis. For the cooling hole analysis, a 1-D compressible-flow network solver is used to determine the internal heat transfer boundary conditions based on metal wall temperatures, flow passage geometric and frictional properties, and coolant flow properties. Recognizing that the metal wall temperature is an input to the coolant flow analysis and the internal heat transfer properties an input to the thermal solid analysis, a coupling routine was necessary. The coupling routine, as shown in Figure 4, iterates between the coolant flow analysis and 3-D thermal solid analysis until the wall temperatures across the heat load surface converge. The wall temperatures are calculated using a steadystate, 3-D finite element thermal solid analysis.

A steady-state finite element thermal solid analysis is conducted to solve the thermal solid solution. The solution is repeated during the cooling hole coupling routine until a converged thermal solid solution is reached. A linearly-elastic steady-state mechanical analysis is conducted subsequent to the thermal solid solution using the thermal solution as input to the temperature-dependent material properties. Therefore, the thermo-mechanical results are obtained in an uncoupled fashion. However, the creep life is calculated as a function of both stress and temperature. The calculation of creep is desired at several sections along the bucket bucket. Section average solid temperatures $(\mathrm{T})$ and stresses $(\mathrm{S})$ in the radial direction were computed from the FEA solutions at eleven sections of interest, identified at $5 \%, 10 \%, 20 \%, 30 \%, 40 \%, 50 \%, 60 \%$, $70 \%, 80 \%, 90 \%$, and $95 \%$ (see Figure 1 ).

The material creep life function used in this study is the OrrSherby-Dorn (OSD) three-term function based on the Arrhenius rate equation ${ }^{2}$. The OSD creep life function gives the time to a specified amount of creep strain as a function of bulk section stress, $\mathrm{S}$, and bulk section temperature, $\mathrm{T}$, and is given as

$$
t_{\text {creep }}=e^{\left(\frac{1}{A+B \ln S}+\frac{\frac{-Q}{R}}{T}\right)}
$$

where the parameters $\mathrm{A}, \mathrm{B}$, and $-\mathrm{Q} / \mathrm{R}$ are empirically determined creep constants corresponding to the time at which the accrual of a pre-defined amount of creep strain is reached. Several tests at various levels of stress and temperature must be performed to determine the creep-strain limit constants. They are unique both to the type of material used as well as the creep strain limit specified. As a result, the creep life at each bucket cross-section of interest can be determined as a function of several upstream variables such as bucket external and internal heat transfer boundary conditions, material properties, geometry, and cooling passage frictional characteristics. Additional material characterization information for creep life modeling of nickel-based super alloys is given by Daleo et al. ${ }^{3,4}$

An Automated Bucket Life Environment (ABLE) was developed to automate the creep life analysis. The environment, depicted in Figure 3, consists of a set of modular PERL scripts run by a single parent PERL script. Each child script automates one of the major analyses. Collectively, they orchestrate the passing of numerous input and output data files between each analysis. Several ANSYS Parametric Design Language (APDL) programs were written to automate the FEA steps including mapping of internal boundary conditions, the parameterization of the cooling-hole diameters, as well as the processing of the section temperatures, stresses, and finally the creep life calculation. A brief description of the RSE/MC method which was executed utilizing the ABLE environment is now given.
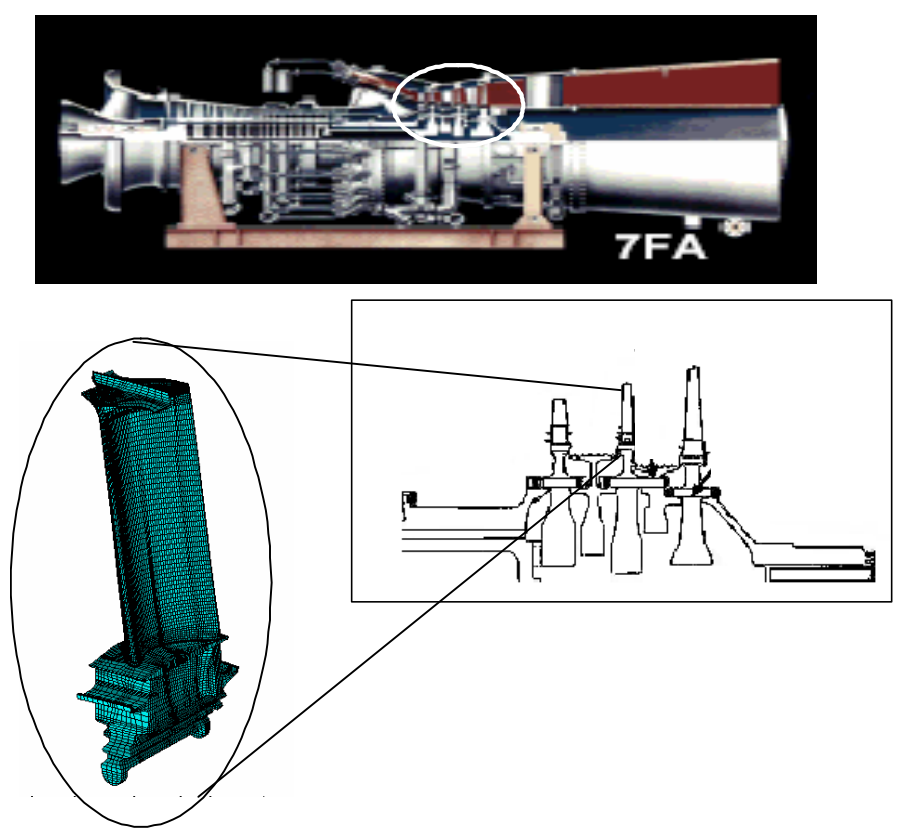

(a)

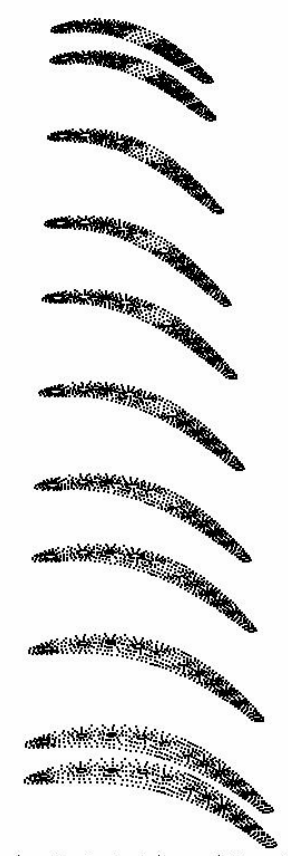

(b)

Figure 1: Turbine airfoil finite element mesh (a) and nodal cross-sections of interest (b). 


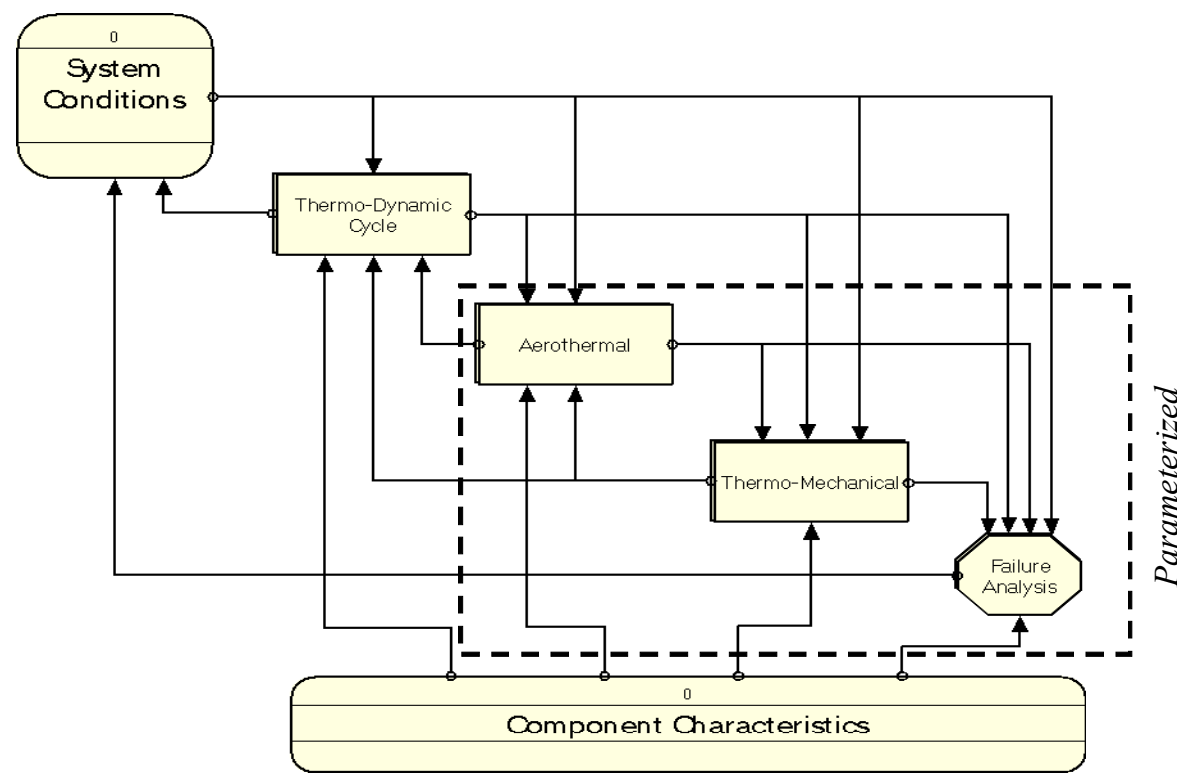

Figure 2: Complex Interrelationship between contributing analyses for conducting part failure assessments.

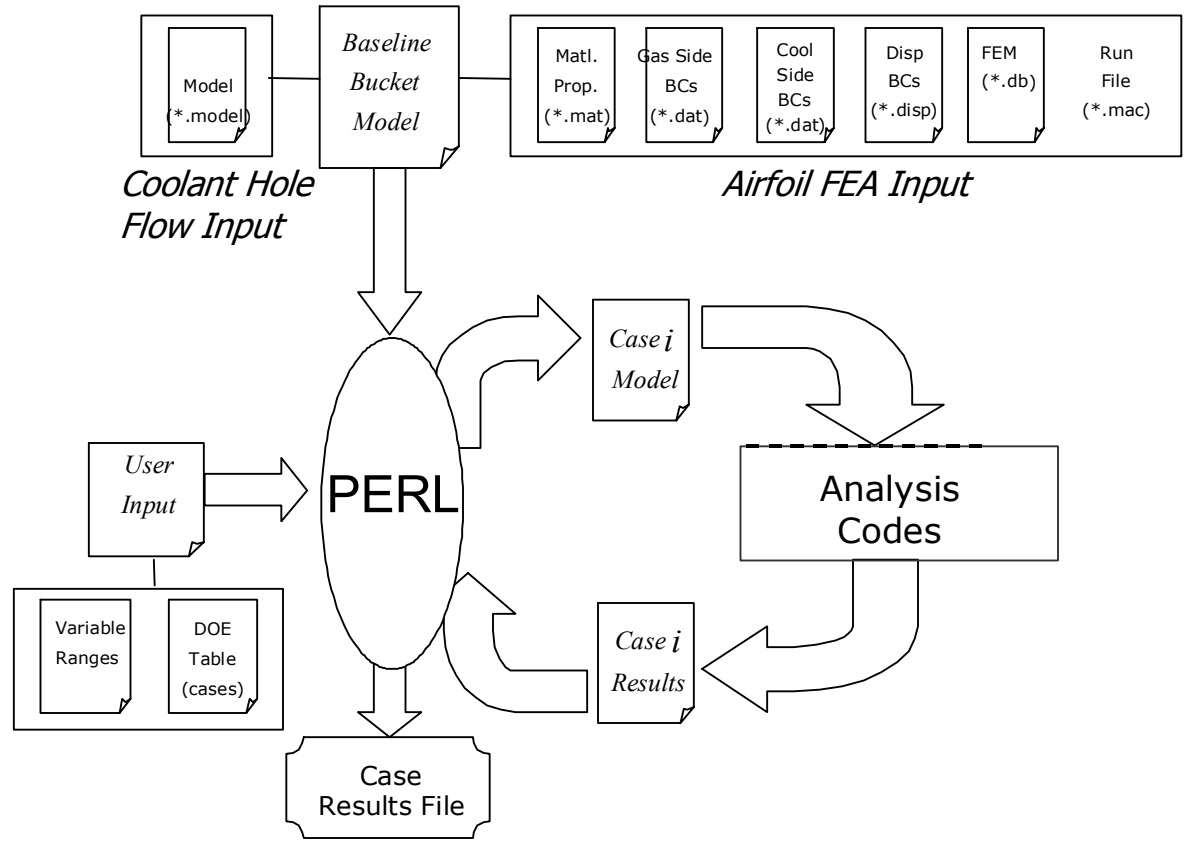

Figure 3: PERL Environment for Linking Analysis Codes 


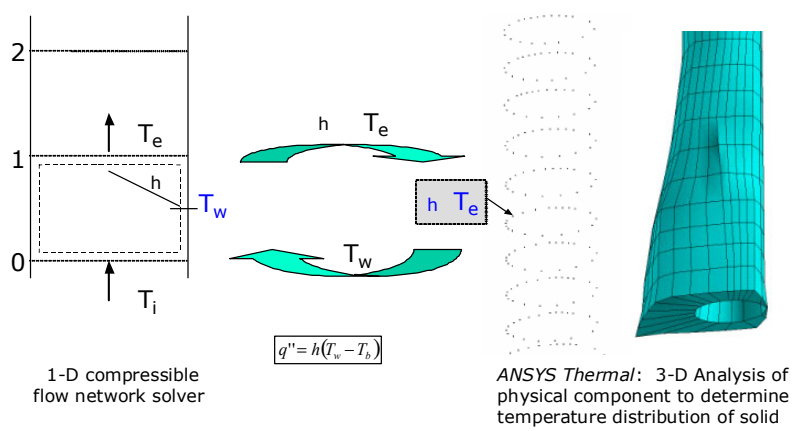

Figure 4: Coupled Cooling Hole Flow and Blade Thermal Solid FEA Analysis

\section{Response Surface Monte Carlo Method}

The Response Surface Method (RSM) method is an applied statistical modeling technique used to create an explicit functional representation of a more complex physical model. The Design-Of-Experiments method (DOE) is employed within the RSM method to select an appropriate combination of variable settings to efficiently sample the actual response space for the regression step. Statistical measures are then taken to gain a more quantitative understanding of the actual response space and potential predictive models of that space. These methods were first introduced by Box and Wilson ${ }^{5}$ and developed to a more useable form by Box and Hunter ${ }^{6}$. A more recent description of RSM is given by Cornell ${ }^{7}$.

The DOE typically used is a three level central composite design (Figure 5). This particular type of DOE permits the modeling of interactions between several of the main factors as well as quadratic main effects.

A representative quadratic, polynomial metamodel commonly used is given as

$$
R=b_{0}+\sum_{i=1}^{k} b_{i} x_{i}+\sum_{i=1}^{k} b_{i i} x_{i}^{2}+\sum_{i=1}^{k-1} \sum_{j=i+1}^{k} b_{i j} x_{i} x_{j}+\varepsilon
$$

where $b_{i}$ are regression coefficients for the 1 st degree terms, $b_{i i}$ are the coefficients for the pure quadratic terms, $b_{i j}$ are the coefficients for the cross-product terms, $\mathrm{x}_{\mathrm{i}}, \mathrm{x}_{\mathrm{j}}$ are the design variables and $\mathrm{x}_{\mathrm{i}} \mathrm{x}_{\mathrm{j}}$ denotes first order interaction between two design variables, and $\varepsilon$ is the error term vector of which its components are assumed to be independent and normally distributed with constant variance. Equation 2 belongs to the class of Response Surface Equations (RSE) which are multivariate closed form expressions that are determined through regression. Through the execution of a balanced DOE, numerous statistical analyses can be performed to check the accuracy, validity, and usefulness of the RSE for both understanding the sampled response or for predictive purposes. The predictive capability of RSEs is paramount to this study since complex, time-consuming analyses can be replaced by an explicit functional relationship between the turbine airfoil life and several life parameters. This is extremely beneficial when probabilistic analyses are conducted since numerous evaluations can be performed over a short amount of time. However, RSEs are applicable to problems with only a few variables. Screening approaches based on DOE or sensitivity analyses can be used to reduce the set of variables to those that are the strongest drivers of the response of interest. Alternatively, RSEs have been applied by Koch et al. ${ }^{8}$ in a hierarchical fashion to create a metamodel of an entire commercial turbofan engine model requiring the use of several variables.

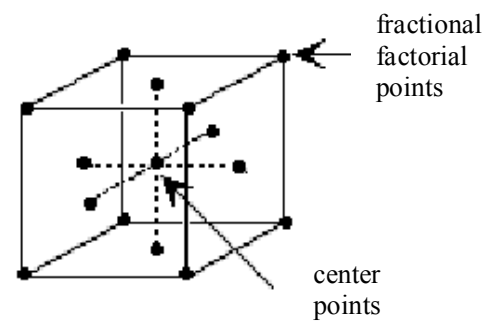

Figure 5: Typical Design of Experiments Sample Point Scheme: Central Composite Design.

Probabilistic simulation analyses of the creep life requiring numerous evaluations of the bucket model are now highly feasible using an RSE metamodel of the life of the gas turbine airfoil. The probabilistic method chosen is the Monte Carlo simulation method (MC) which is the most general and most accurate probabilistic method given enough simulations. The $\mathrm{RSM} / \mathrm{MC}$ method can model not only the variation of the control variables, but their underlying statistical distribution and joint randomness as well. Therefore, the combined $\mathrm{RSM} / \mathrm{MC}$ method is considered an accurate probabilistic realization of the creep life of the component in question

\section{Results}

Initially, sixteen potential creep life variables were chosen based on engineering experience and judgement. Since over a dozen variables were identified, it was deemed necessary to reduce the number of variables under consideration. This was accomplished using a DOE screening study. A 2-level fractional factorial DOE requiring 30 cases was executed and the response for each case evaluated. Using Analysis of Variance (ANOVA) ${ }^{9}$, the relative contribution to the variation of creep life was quantified. The result of this step is shown in Figure 6. Notice that five variables were found to be the primary contributors to creep while the remaining variables were found to be relatively insignificant. These five variables contribute to more than $80 \%$ of the variation of creep life over the ranges of the sixteen variables initially considered.

The five primary variables include the external hot gas temperature field (SEXTT), external heat transfer coefficient field (SEXTh), creep constant variation parameter $(\mathrm{CN})$, friction factor multiplier for cooling holes 2 through 5 (SFM25), and the friction factor multiplier for cooling holes 6 through 8 (SFM68). The control variables SFM25 and SFM68 are scalar values that operate on a one-dimensional field of internal cooling hole friction factor values for cooling holes 2 through 5 and 6 through 8 , respectively. The friction factor multiplier variable for the first cooling hole was determined through simulation to be insignificant for the range of the 
variables considered. SEXTh and SEXTT are scalar values that represent a 3-D field of convective heat transfer coefficients and gas adiabatic wall temperatures for the external surface of the bucket, respectively. The $\mathrm{CN}$ parameter is an additional constant added to the exponential term in the creep life expression as follows

$$
\left.t_{\text {creep }}=e^{\left(\frac{1}{A+B \ln S}+\frac{\frac{-Q}{R}}{T}+C N \cdot S c\right.}\right)
$$

where the $C N$ parameter represents a composite variation of the three creep constants, $\mathrm{A}, \mathrm{B}$, and $-\mathrm{Q} / \mathrm{R}$ and the $S c$ parameter is a material specific constant determined through specimen testing. The appropriate range of each variable was also chosen using a combination of engineering judgement and available data. The remaining variables were set to their expected, baseline value.

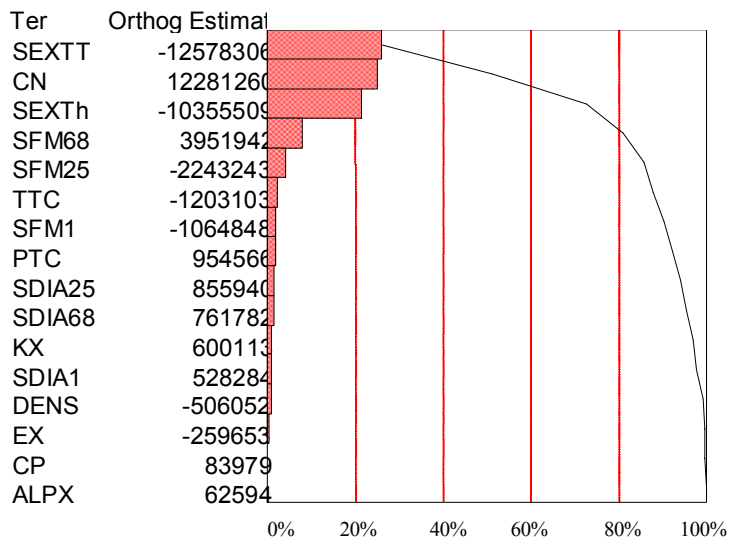

Figure 6: Pareto plot showing contributing creep life factors

The next step in the process involved creating a higher resolution DOE using the five variables selected during the screening process. A 3-level, resolution 4, fractional-factorial, central-composite DOE was created, which required 43 bulk creep life evaluations. The levels chosen were high, medium, and low corresponding to the $-3 \sigma$, mean, and $+3 \sigma$ values for each of these five variables. These cases included one center point where all the variables were set to their mean expected value. The other variables that were determined to be negligible still were required during the analysis for each of these cases. Their values were set to their expected mean value throughout all 43 evaluations.

Least squares regression was applied to fit a quadratic response surface equation (RSE) to the bulk creep life data from the DOE sample points previously determined. A goodness of fit parameter known is the coefficient of determination, or $\mathrm{R}^{2}$, is used as a means of assessing the accuracy of the model in predicting the values of the sampled points. The $\mathrm{R}^{2}$ for this step was found to be 0.914396 . Although for experimental studies this would be acceptable, it is not so for response values determined using a deterministic model providing a unique solution. The statistical assessment of the bulk creep life DOE points suggest that a highly non-linear relationship exists between the variables and the bulk creep life. This is explained physically since creep life tests of this class of material follow an exponential function ${ }^{4}$ (reference equation 1 ).

Since the quadratic function didn't approximate the data properly, another functional form was pursued. The problem was to determine the functional form of the approximator. In this case the selection of the proper function is trivial. The bulk creep life is exponential and therefore a logarithmic transformation function was chosen. The quadratic response approximation was fit to the logarithm of the actual bulk creep life, known as a dependent variable transformation, using the life values already determined. Not surprisingly, this proved to be highly accurate in predicting the log of bulk creep life behavior. The $\mathrm{R}^{2}$ was perfect being very close to unity. Assumptions of zero mean error and constant error variance of the model are were also verified to ensure that the necessary statistical model assumptions were met. The predicted values must be transformed back to the life scale to to assess the actual accuracy of the model and make useful life predictions. The maximum error of the fitted model after transforming the predicted logarithmic lives back to actual creep lives was $0.4 \%$. The mean error was found to be $7.24 \mathrm{E}-7 \%$. Hence, the error, although amplified by the exponential transformation, is still minimal.

Finally, the model had to be tested over numerous points across the spectrum of possible variable value combinations to ensure that the transformed model was an accurate general approximation of the bulk creep life. Thirty randomly generated cases were executed to do this. The maximum error between the predicted versus actual bulk creep life over all 73 cases was still $0.6 \%$ with a mean error of $0.03 \%$, suggesting that the approximation is an accurate representation of the bulk creep life.

Once the accuracy and validity of the response surface equation is verified, several useful activities can be carried out using this equation. Given that the closed-form approximation, or response surface equation (RSE), is an accurate representation of the more complex physical model, it can be utilized by simulation approaches to generate a population of bulk creep lives necessary to determine its probabilistic characteristics. The Monte Carlo method was then applied to conduct the probabilistic analyses using the RSE generated. The analysis consisted of choosing the statistical characteristics of the five primary random variables selected during the prior screening study. The variable characteristics used in this study are given in Table 1, where COV stands for the coefficient of variation which is defined as the ratio of the standard deviation and the mean. The COV of the friction factor parameters was set to a very large value representing considerable scatter in the friction characteristics of the blade cooling holes as compared to the other parameters. Actual measured cooling hole flow rate data was available that suggested such a high variation. This was accounted for using the friction factor multipliers variables SFM25 and SFM68. Each parameter (Table 1) was assumed to 
follow a normal distribution with constant mean and variance. In addition, the variables were considered to be statistically independent of each other. A total of 100,000 simulations were run to generate an accurate estimation of the characteristics of the resulting bulk creep life distribution. Rather than run the complete and time-consuming physical model, the previously generated RSE was used instead. This approach was quite efficient considering that 100,000 simulations of the response surface equation took only a few minutes of CPU time versus months even years had the original physical model been used.

Table 1: Parameter Statistics

\begin{tabular}{|l|ccc|}
\hline Random Variable & Distribution & Mean & COV \\
\hline CN & normal & 0 & 1 \\
SEXTh & normal & nominal & $5 \%$ \\
SEXTT & normal & nominal & $0.77 \%$ \\
SFM25 & normal & nominal & $60 \%$ \\
SFM68 & normal & nominal & $75 \%$ \\
\hline
\end{tabular}

The result of the Monte Carlo Simulation in the form of a probability density function is given in Figure 7, with the best fit lognormal and Weibull parameters found using maximum likelihood theory. The resulting distribution is, notsurprisingly, lognormal; which follows bulk creep life experience and can also be proven mathematically using analytical statistics. The best-fit Weibull distribution is obviously (see Figure $7 \mathrm{~b}$ ) inferior to the lognormal distribution for modeling this data set.

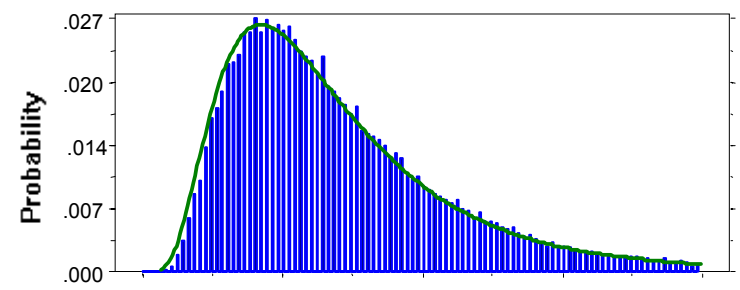

(a)

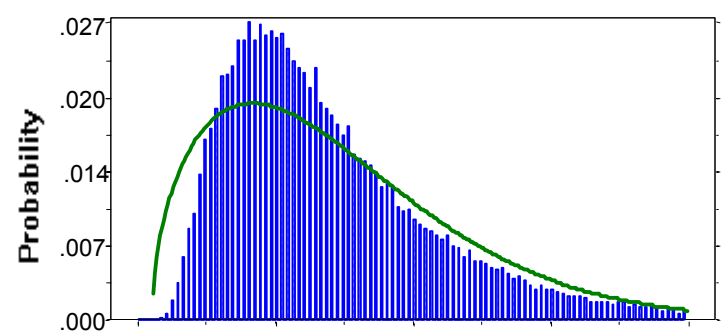

(b)

Figure 7: Lognormal (a) and Weibull (b) Distributions Fit to the Monte Carlo Simulation Life Data.

A useful result of the Monte Carlo simulation is the probabilistic calculation of the sensitivity of the bulk creep life to each of the individual parameters. Figure 8 shows the relative sensitivity of the bulk creep life with respect to each parameter. The sensitivity was calculated by normalizing the square of the correlation coefficients between the bulk creep life and each of the variables. As expected, the bulk creep life variation is most sensitive to the external gas field parameters and the creep constant variation. The sensitivity analysis results in the same ranking as that determined using the Pareto analysis during the design of experiment study. However, the major difference between the two methods is that the DOE Pareto sensitivity method uses only the response values of a few pre-selected DOE cases to calculate the relative sensitivities; while the probabilistic sensitivity calculation uses the entire population of bulk creep lives produced during the Monte Carlo simulation. Unlike the Pareto plot, the probability sensitivity calculation using Monte Carlo is a function of not only the deterministic model used but also the distributions of the life variables. Therefore, non-normal input variable distributions could produce a different sensitivity result.

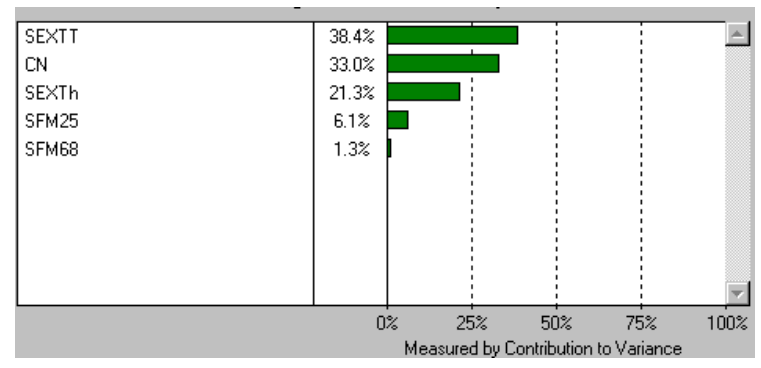

Figure 8: Probabilistic Sensitivity Indices.

Another important result of such a probabilistic analysis is the determination of the life corresponding to a low probability of failure. Such an approach is used to ensure that the majority of the distribution lies beyond the intended useful life of the component. However, this tail-end of the probabilistic distribution can be quite sensitive to both the probabilistic method used, as well as the variables considered in the analysis. The global sensitivity of the probabilistic distribution with respect to considering more and more variables is shown in Figure 9. Each of the five dominant parameters was added sequentially and the Monte Carlo simulation repeated for each case. Notice that both the location and scale of the distribution are affected by the choice of random variables. The lowprobability region of the cumulative distribution function history is given in Figure 10 to illustrate just how sensitive it is to the variables considered. Interestingly, the distribution appears to stabilize as all of the five dominant parameters are considered. This gives credence to the decision made to screen out other variables since their consideration will likely not vary the resulting low probability of failure life by much. Reducing the dimensionality of probabilistic analyses is important when considering those of complex components requiring longrunning analyses. 


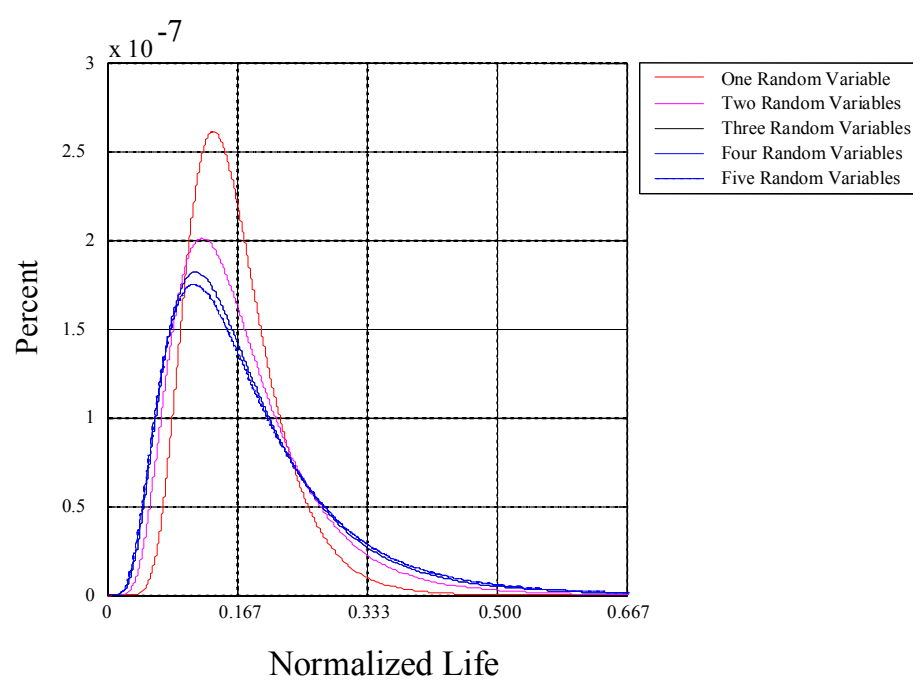

Figure 9: Probability Density Functions Generated As More and More Variables are Considered.

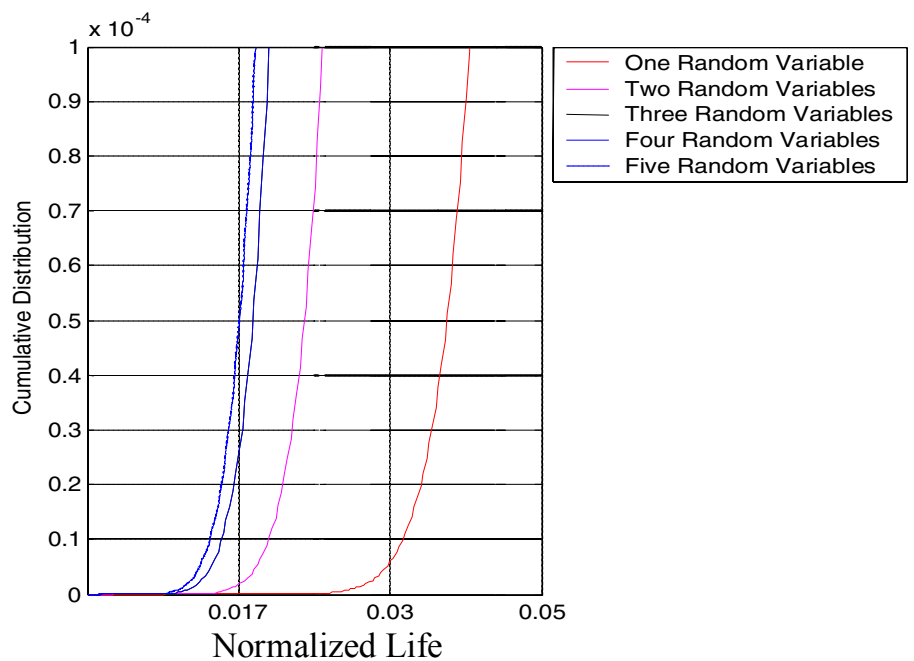

Figure 10: Cumulative Distribution Functions Generated As More and More Variables are Considered.

A more rigorous analytical solution can be obtained using classical statistics. The analytical class of probabilistic methods uses first and second-order approximations of the deterministic response and probability density function integral. Approximations are generally determined using power series expansion such as the following Taylor series expansion about the mean input vector, $\mathrm{X}$, given as

$Z=g(X)=g\left(x_{1}, x_{2}, \ldots, x_{n}\right)$

and

$$
Z \approx g(\bar{X})+\sum_{i=1}^{n} \frac{\partial g}{\partial X_{i}}(X-\bar{X})+\frac{1}{2} \sum_{i=1}^{n} \sum_{j=1}^{n} \frac{\partial^{2} g}{\partial X_{i} \partial X_{j}}(X-\bar{X})(X-\bar{X})+\ldots
$$

where $\bar{X}$ is the vector of variable values at a suspect point which is usually the mean vector. The Taylor series expansion of the probability integral was pursued as follows.
By taking the logarithm of equation 4 and setting the variables to their mean values a transformed response is given as

$$
Y=\ln N=\frac{1}{A+B \ln (S)}+\frac{A C T}{T+460}+C N * S c
$$

The location of the resulting lognormal distribution can be calculated as

$\mu_{Y}=\mu_{\ln N} \approx \frac{1}{A+B \ln \left(\mu_{S}\right)}+\frac{A C T}{\mu_{T}+460}$

where each random variable is set to its mean value. The mean of $\mathrm{CN}$ is zero for this application so the third term drops out However, the calculation of the variance of the bulk creep life is not as simple. The first term of a Taylor series expansion can be used to approximate the complex variance integration as

$\sigma_{\ln (N)}^{2}=V[Y]=\left(\frac{d Y}{d x_{1}}\right)^{2} \cdot \sigma_{x_{1}}^{2}+\cdots+\left(\frac{d Y}{d x_{n}}\right)^{2} \cdot \sigma_{x_{n}}^{2}$

Performing this expansion the mean vector and computing the individual partial derivatives yields the following result

$\sigma_{\ln (N)}=\sqrt{\left(\left[A+B \cdot \ln \left(\mu_{S}\right)\right]^{-4} \cdot B^{2} \cdot \frac{1}{\mu_{S}^{2}}\right) \cdot \sigma_{S}^{2}+\left(\left[\mu_{T}+460\right]^{-4} \cdot A C T^{2}\right) \cdot \sigma_{T}^{2}+S c^{2}}$

The mean and variance of each input variable were the same as those specified at the start of this study. The mean stress and temperature were determined from the baseline analysis which was conducted with each of the five primary variables to their respective mean. The resulting lognormal distribution using the analytical approach is plotted in Figure 11 superimposed over the distribution produced using the RSE method.

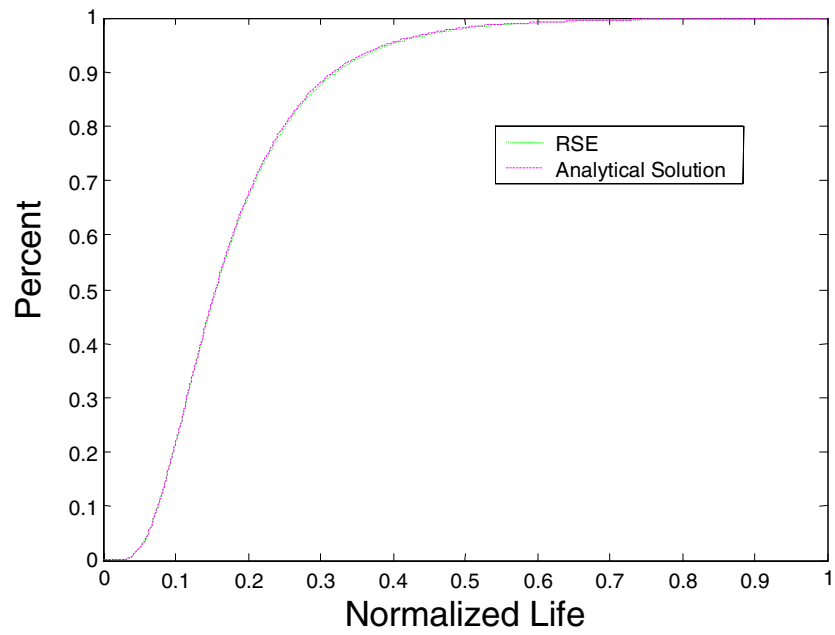

Figure 11: Comparison of both probabilistic methods.

It is apparent that the RSE/MC method agrees very well with the analytical solution. Hence, one would conclude that the analytical method is far superior to the RSE/MC result in terms of the amount of required statistical knowledge and execution time, especially since the analytical solution required only one evaluation of the response. However, this method was used solely for validation purposes. Further, the method is limiting in that it assumes that the input random variables are independent and symmetrically distributed. Should one venture from this condition then the RSE/MC method would quickly 
become more appealing. Interestingly, Wallace and Mavris ${ }^{10}$ quantified such local variable statistical behavior for a similar component in an airborne gas turbine and found that strong correlation exists amongst parameters such as core and coolant flow temperature. Further, they conducted a Bera-Jarque ${ }^{11}$ test for normality which determined with significant evidence that many of the local parameters were in fact non-normally distributed. This finding is of particular relevance to this study so an initial yet partial consideration of variable joint randomness was performed.

Joint randomness and distribution information of the primary input variables was not available during this study. Instead, a sample case was pursued to illustrate the effect of neglecting such conditions. It can be argued that the heat transfer coefficient and gas temperature fields are at least moderately and positively correlated. A correlation coefficient of +0.5 was assumed for this variable pair and the RSE/MC method reexecuted. The resulting distribution is compared to the original distribution in Figure 12. The distribution produced with the assumed correlation has greater dispersion. Considering that positive correlation between these variables is to be expected, the original low probability result is therefore not conservative. On the whole, the variation of the life across the entire distribution is insignificant; however, for low-probability cases (important to reliability engineers) the result is surprising. Future work is recommended to quantify the local input variable statistical behavior to improve the probabilistic assessment of such components.

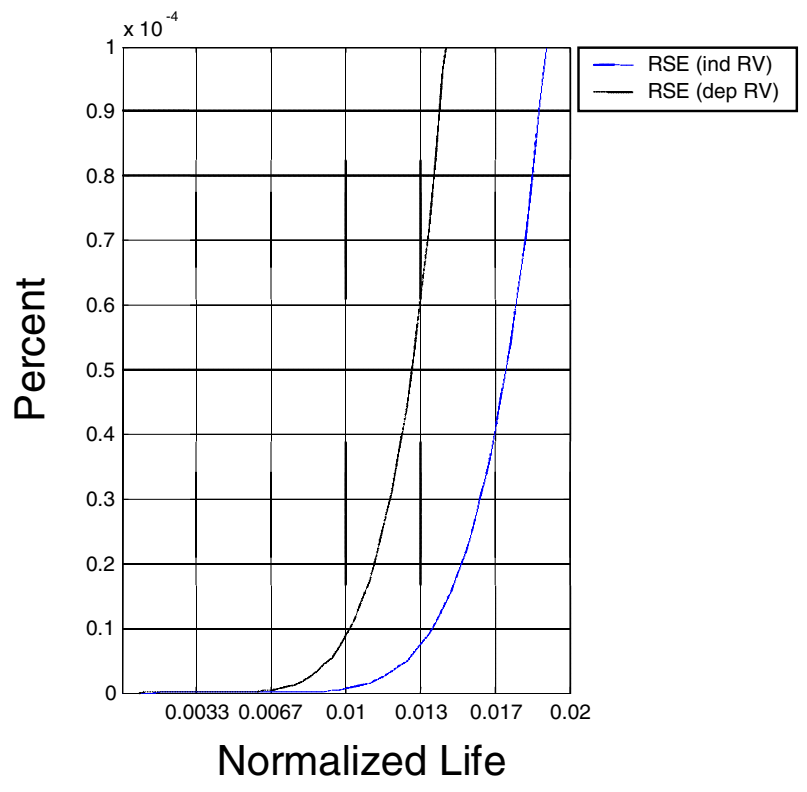

Figure 12: Effect of joint randomness on response distribution.

\section{Conclusions}

Using a screening DOE study of a complex airfoil thermomechanical simulation only five of sixteen variables were found to be strong contributors to the calculated creep life. A highly accurate metamodel (RSE) of the time-consuming gas turbine airfoil creep life model was then created as a function of these primary variables. The RSE created was successfully used in a Monte Carlo simulation to perform the necessary probabilistic analysis. The part life under bulk creep failure was found to be lognormally distributed and was validated via a solution determined using analytical statistics. Due to the symmetry of the input variable distributions, the ranking of the primary input parameters using probabilistic sensitivity indices resulted in the same order of importance as that of a Pareto ranking using statistical DOE sampling. The effect of successfully considering additional parameters on the resulting distribution was explored suggesting that the composite response distribution can be found rapidly using solely the primary variables selected during the screening exercise as opposed to the entire variable set. Finally, a simple exercise of introducing input parameter dependency was conducted suggesting that neglecting variable dependency in a part life probabilistic study can lead to non-conservative low-probability predictions. Future work is recommended to develop and apply methods to characterize the local parameter statistical space for improving such probabilistic part life predictions.

\section{Acknowledgments}

The authors would like to thank General Electric Power Systems for their continued support of this project. Dr. Ian Hunter of General Electric Power Systems is to be especially thanked for his involvement with the original industry sponsored study. Dr. Zhimin Liu, Mrs. Rhenwua Wang, and Dr. Vitali Volovoi of the Georgia Tech Aerospace Systems Design Laboratory are to be thanked for their assistance.

\section{References}

1. Haldar, A., and Mahadevan, S., 2000, Probability, Reliability and Statistical Methods in Engineering Design, John Wiley and Sons, Inc, New York, NY.

2. Orr, R.L., Sherby, O.D., and Dorn, J.E., 1954, "Correlations of rupture data for metals at elevated temperatures", Trans. ASM, 46, 113.

3. Daleo, J.A., Ellsion, K.A., and Woodford, D.A., 1999, "Application of Stress Relaxation Testing in Metallurgical Life Assessment Evaluations of GTD111 Alloy Turbine Buckets," Journal of Engineering for Gas Turbines and Power, Vol. 121, pp. 129-137.

4. Daleo, J.A. and Wilson, J.R., 1999, "GTD111 Alloy Material Study," Journal of Engineering for Gas Turbines and Power, Vol. 120, pp. 375-382.

5. Box, G.E.P. and Wilson, K.B., 1951, "On the Experimental Attainment of Optimum Conditions." Journal of Royal Statistical Society, B13, 1-38, Discussion 38-45. 
6. Box, G.E.P., Hunter, W.G., and Hunter, J.S. 1978. Statistics for Experimenters: An Introduction to Design, Data Analysis, and Model Building. New York: Wiley and Sons, Inc.

7. Cornell, John A., 1990, "How to Apply Response Surface Methodology." ASQC Basic References in Quality Control: Statistical Techniques, 2nd edition, American Society for Quality Control.

8. Koch, P.N., Mavris, D.N., Mistree, F., 1998, "Multi-Level, Partitioned Response Surfaces for Modeling Complex Systems," Presented at the 7th AIAA/USAF/NASA/ISSMO Symposium on Multidisciplinary Analysis and Optimization, St. Louis, MO, September 2-4.

9. Scheffé, H. 1959, The Analysis of Variance, John Wiley \& Sons, New York.

10. Wallace, J.M., and Mavris, D.M., 2002, "Propulsion System Reliability Prediction and Optimization Using a Multi-Physics Environment," AIAA/ISSMO Symposium on Multidisciplinary Analysis and Optimization, Atlanta, GA.

11. Bera, A.K. and Jarque, C.M. 1982. "Model Specification Tests: a Simultaneous Approach", Journal of Econometrics, v. 20, pp. 59-82. 